11 Short Title: In situ structure of the pKM101-encoded T4SS

\title{
23 Keywords:
}

24 cryoelectron tomography, DNA conjugation, type IV secretion, Pilus, protein transport 


\section{ABSTRACT}

28 Bacterial conjugation systems are members of the type IV secretion system (T4SS) superfamily. T4SSs can be

29 classified as 'minimized' or 'expanded' based on whether they are composed of a core set of signature subunits or

30 additional system-specific components. Prototypical 'minimized' systems mediating Agrobacterium tumefaciens

31 T-DNA transfer and pKM101 and R388 plasmid transfer are built from subunits generically named VirB1-

32 VirB11 and VirD4. We visualized the pKM101-encoded T4SS in the native cellular context by in situ

33 cryoelectron tomography (CryoET). The $\mathrm{T}_{\mathrm{SSS}} \mathrm{PKM} 101_{1}$ is composed of an outer membrane core complex (OMCC)

34 connected by a thin stalk to an inner membrane complex (IMC). The OMCC exhibits 14-fold symmetry and

35 resembles that of the $\mathrm{T}_{4} \mathrm{SS}_{\mathrm{R} 388}$ analyzed previously by single-particle electron microscopy. The IMC is highly

36 symmetrical and exhibits 6-fold symmetry. It is dominated by a hexameric collar in the periplasm and a

37 cytoplasmic complex composed of a hexamer of dimers of the VirB4-like TraB ATPase. The IMC closely

38 resembles equivalent regions of three 'expanded' T4SSs previously visualized by in situ CryoET, but differs

39 strikingly from the IMC of the purified $\mathrm{T}_{4} \mathrm{SS}_{\mathrm{R} 388}$ whose cytoplasmic complex instead presents as two side-by-side

40 VirB4 hexamers. Analyses of mutant machines lacking each of the three ATPases required for T4SS $\mathrm{pKM}_{\mathrm{pK} 101}$

41 function supplied evidence that $\mathrm{TraB}_{\mathrm{B} 4}$ as well as VirB11-like TraG contribute to distinct stages of machine

42 assembly. We propose that the VirB4-like ATPases, configured as hexamers-of-dimers at the T4SS entrance,

43 orchestrate IMC assembly and recruitment of the spatially-dynamic VirB11 and VirD4 ATPases to activate the

44 T4SS for substrate transfer.

\section{SIGNIFICANCE}

47 Bacterial type IV secretion systems (T4SSs) play central roles in antibiotic resistance spread and virulence. By

48 cryoelectron tomography (CryoET), we solved the structure of the plasmid pKM101-encoded T4SS in the native

49 context of the bacterial cell envelope. The inner membrane complex (IMC) of the in situ T4SS differs remarkably

50 from that of a closely-related T4SS analyzed in vitro by single particle electron microscopy. Our findings

51 underscore the importance of comparative in vitro and in vivo analyses of the T4SS nanomachines, and support a

52 unified model in which the signature VirB4 ATPases of the T4SS superfamily function as a central hexamer of

53 dimers to regulate early-stage machine biogenesis and substrate entry passage through the T4SS. The VirB4 
54 ATPases are therefore excellent targets for development of intervention strategies aimed at suppressing the action

55 of T4SS nanomachines.

\section{INTRODUCTION}

58 Many species of bacteria deploy type IV secretion systems (T4SSs) to deliver DNA or protein substrates to target 59 cells (1-3). T4SSs designated as 'minimized' systems are assembled from a core set of signature subunits, while 60 others termed 'expanded' are compositionally and structurally more complex, possibly reflecting adaptations

61 arising over evolutionary time for specialized functions (3). In Gram-negative species, 'minimized' systems are

62 assembled from 12 subunits named VirB1 - VirB11 and VirD4 based on the paradigmatic Agrobacterium tumefaciens VirB/VirD4 T4SS (3). Three subunits (VirB7, VirB9, C-terminus of VirB10) assemble as an outer membrane core complex (OMCC) that spans the distal region of the periplasm and outer membrane (OM) (4). Four integral membrane components (VirB3, VirB6, VirB8, N-terminus of VirB10) and two or three ATPases (VirB4, VirD4, +/- VirB11) together comprise the inner membrane complex (IMC) $(5,6)$. Some T4SSs elaborate an extracellular organelle termed the conjugative pilus from homologs of the VirB2 pilin and VirB5 pilus-tip subunit (3). 'Expanded' systems are composed of homologs or orthologs of most or all of the VirB/VirD4 subunits plus as many as 20 components that are system-specific (3).

To better understand the mechanism of action of T4SSs and the structural bases underlying functional diversity of this translocation superfamily, there is growing interest in solving the structures of intact machines and machine subassemblies. OMCCs are generally stable and amenable to purification, and structures are now available for

74 OMCCs from several 'minimized' and 'expanded' systems at resolutions approaching $3 \AA$ (4, 6-12). Structural 75 analyses of inner membrane (IM) portions of T4SSs have been considerably more challenging due to problems of 76 instability and dissociation during purification. Presently, one structure exists for a 'minimized' system encoded

77 by the conjugative plasmid R388. Designated the $\mathrm{VirB}_{3-10}$ complex, this structure was obtained by overproduction 78 of the VirB3 - VirB10 homologs, affinity purification of the detergent-solubilized complex, and analysis by 79 negative-stain electron microscopy (nsEM) (6). The $\operatorname{VirB}_{3-10}$ complex consists of the OMCC and IMC connected 80 by a thin, flexible stalk. The IMC is composed of a highly asymmetric IM platform connected to two side-by-side 
81 hexamers of the VirB4 ATPase extending into the cytoplasm. In an updated structure, two dimers of the VirD4

82 ATPase were shown to integrate between the VirB4 barrels (13).

84 IMCs of 'expanded' T4SSs have not yet been analyzed by single-particle EM. However, recent advances using in 85 situ cryoelectron microscopy (CryoET) have enabled visualization of the Legionella pneumophila Dot/Icm,

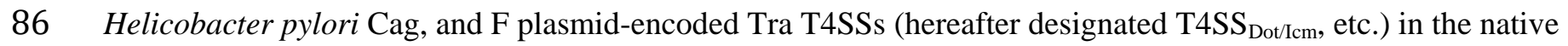

87 context of the cell envelope (14-21). Remarkably, in contrast to the IMC of the VirB $3-10$ structure, the IMCs of all

88 three 'expanded' systems clearly exhibit 6-fold symmetry and the VirB4 ATPases assemble as a central hexamer

89 of dimers at the channel entrance (16-18).

91 Here, we solved the in situ structure of the pKM101-encoded T4SS, which is phylogenetically (Fig. S1A) and

92 functionally closely related to the R388-encoded T4SS, to the extent that the two 'minimized' systems can

93 translocate each other's plasmids and some machine subunits are exchangeable (8). We report that the IMC of the

94 in situ $\mathrm{T}_{4} \mathrm{SS}_{\mathrm{pKM} 101}$ adopts the 6-fold symmetry observed for the equivalent regions of the 'expanded' T4SSs. Most

95 strikingly, the VirB4 homolog $\operatorname{TraB}\left(\operatorname{TraB}_{\mathrm{B} 4}\right)$ is arranged as a central hexamer of dimers, not the side-by-side

96 hexameric barrels visualized for this ATPase in the purified $\operatorname{VirB}_{3-10}$ complex. Mutant machines lacking $\operatorname{TraB}_{\mathrm{B} 4}$ or

97 VirB11-like TraG exhibit structural differences in the IMC compared with the wild-type machine suggestive of

98 contributions of these ATPases to distinct stages of $\mathrm{T} \mathrm{SS}_{\mathrm{pKM} 101}$ machine assembly. Together, our findings support

99 a model in which the VirB4 ATPases, configured as central hexamers of dimers at the bases of T4SSs, play key

100 roles in several early-stage morphogenetic reactions required for machine biogenesis.

102 RESULTS AND DISCUSSION

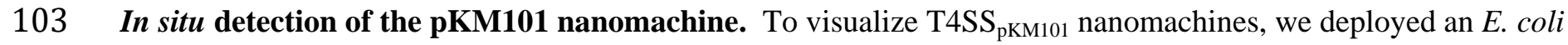

$104 \operatorname{mreB} \min C$ mutant carrying pKM101 to generate small ( $<300 \mathrm{~nm}$ in diameter) minicells (17). Minicells are ideal

105 for in situ CryoET because of their small size and full metabolic capacity (22), including the ability to deliver

106 plasmids such as F (17) or pKM101 (Fig. S1B) through encoded T4SSs to recipient cells. We used a high-

107 throughput CryoET pipeline to visualize thousands of E. coli minicells (see Fig. S2 for workflow). The pKM101 
108 nanomachines were smaller and more difficult to detect than the F plasmid-encoded T4SS or other 'expanded'

109 systems we have previously characterized (16-18), but we were able to detect pKM101-encoded structures among

110 every 2 or 3 minicells examined (Figs. 1Ai-iv, Movie S1). Importantly, minicell preparations from the parental

111 strain UU2834 alone lack these surface structures, confirming that the presence of pKM101 in the host strain is

112 required for their elaboration.

114 The pKM101-encoded structures consist of periplasmic cone-shaped complexes near the outer membrane (OM)

115 without or with associated thin 'stalk' structures extending to the IM (Figs. 1Ai, iii). OMCCs lacking stalk

116 structures represented about half of the initially picked particles, but likely represent assembly intermediates or

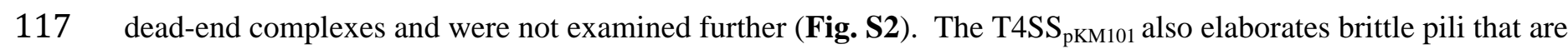

118 readily detached or sloughed from cells (23). We detected some pKM101-encoded pili, bound either to the

119 OMCC-stalk structures or to sites on the OM devoid of underlying basal densities (Figs. 1Ai, ii, S3). Because the

120 pKM101 pili were rarely detected, we focused on solving the in situ structure of the cell-envelope-spanning

121 nanomachine to allow for comparisons with other T4SS structures solve in situ or in vitro $(4,6,7)$.

123 Visualization of the in situ OMCC. From 287 nanomachine subtomograms extracted from 560 tomographic

124 reconstructions, we generated in situ structure of the OMCC at a resolution of $\sim 37 \AA$ (Figs. 1B, S2). Three-

125 dimensional classifications revealed 14-fold symmetrical features of the OMCC, which were resolved further by

126 imposing a 14-fold symmetry during refinement (Fig. S2). In the refined structure, the OMCC is clearly seen

127 attached to the OM where it causes an invagination of the outer leaflet (Figs. 1Bi, ii). The upper region,

128 designated as the O-layer (4), is $185 \AA$ wide and $70 \AA$ in height. In side-view, the complex forms at least two

129 contacts with the OM, the first mediated by a central cap and the second by the periphery of the OMCC (Fig.

130 1Bii). In the middle of the central cap and extending across the OM is a region of lower density that might

131 correspond to the OM-spanning channel. In top-down view, the periphery of the OMCC clearly consists of 14

132 knobs arranged in a ring of $\sim 185 \AA$ in width (Fig. 1Biii). The knobs are connected via spokes to a central

133 contiguous ring that conforms to the base of the cap. In 3D renderings, it is evident that the 14 peripheral knobs

134 interact with the OM (Fig. 1C). Notably, besides invagination of the OM at the cap junction, the region of the OM 
135 between the central cap and peripheral contacts lacks an inner leaflet, suggesting that the OM undergoes extensive

136 remodeling during machine biogenesis (Figs. 1Bii, C).

138 The O-layer chamber is closed at the OM junction and widens to $\sim 150 \AA$ where it joins the lower region of the

139 OMCC known as the I-layer (4). The I-layer has a height of $65 \AA$ and is slightly wider than the O-layer, although

140 the outer boundary of the O-layer is blurred because of density contributed by the peptidoglycan (PG) layer (Fig.

141 1Bii). The I-layer narrows at its base where the central chamber has a diameter of $\sim 70 \AA$. A stalk density embeds

142 into the central cavity and projects through the periplasm to the IM (Fig. 1Bi, ii, C). Overall, the in situ OMCC

143 structure has a total height of $135 \AA$ (Fig. 1Bii).

145 Although the resolution of the visualized OMCC $(\sim 37 \AA)$ is lower than that achieved by single particle analyses

$146(<20 \AA)(4,24)$, the in situ and in vitro OMCCs exhibit 14-fold symmetry and have similar cross-section

147 dimensions of $185 \AA$ A (Fig. S4). They are also composed of distinct O- and I-layers that house large central

148 chambers $(4,7)$. The in vitro structure, however, is more elongated ( $185 \AA$ in height) than the in situ structure

149 (135 ̊) (Fig. S4B, C). The O-layer and the upper portion of the I-layer are intrinsically stable due to extensive

150 networks of interactions between the $\mathrm{TraF}_{\mathrm{B} 10}$ and $\mathrm{TraO}_{\mathrm{B} 9}$ constituents (24). By contrast, the lower portion of the I-

151 layer, which is built from $\alpha$-helical linker domains of $\operatorname{TraF}_{\mathrm{B} 10}$ that connect the OMCC to the IM, is highly flexible

$152(4,24)$, Therefore, we suspect that the region of the OMCC that resolves well in the in situ structure corresponds

153 to the O-layer and upper portion of the I-layer, whereas the linker domains comprising the lower portion of the I-

154 layer are either too flexible for detection in vivo or they fold inward to form part of the central stalk.

155 Gratifyingly, an X-ray structure of the O-layer (7) fits well into the O-layer of the in situ structure (Fig. S4D). The

156 OMCC of the in vitro VirB $_{3-10}$ complex also generally superimposes well onto the equivalent subassembly of the

157 in situ $\mathrm{T}_{4} \mathrm{SS}_{\mathrm{pKM} 101}$, although the latter structure has additional densities at the top comprising the peripheral OM

158 contacts and laterally that might correspond to associated PG (Fig. S4Ei - iii), The convergence of OMCC

159 architectures from the R388 and pKM101 systems is in line with previous findings that the OMCC from the R388

160 machine can be swapped for that of the pKM101 system to yield a functional chimeric system (8). 
162 Although a flexible stalk connecting the OMCC and inner membrane complex (IMC) was previously visualized in

163 the $\operatorname{VirB}_{3-10}$ complex (6), at the time it was not known if the stalk corresponded to a central channel that was

164 structurally distorted during detergent solubilization of the nanomachine (Fig. S4Ei, iii). Here, our finding that a

165 stalk density (Fig. S4iii) lacking a discernible channel joins the OMCC to the IMC in the in situ T4SS ${ }_{\mathrm{pKM} 101}$

166 confirms that the stalk is a prominent feature of 'minimized' systems. This distinguishes the 'minimized' systems

167 from 'expanded' systems such as the F plasmid Tra and L. pneumophila Dot/Icm T4SSs (16, 17), whose in situ

168 structures clearly possess central channels bridging the IMC and OMCC subassemblies (Figs. S4Eiii, Fi-iii). It is

169 also interesting to note that the central stalk of the $\mathrm{VirB}_{3-10}$ structure spans a gap of $\sim 33 \AA$ between the OMCC and

170 IMC. Here, however, we observed that the gap between the OMCCs and IMCs of different T4SS ${ }_{\mathrm{pKM} 101}$ machines

171 was more variable and in the range of $\sim 50-70 \AA$. In situ CryoET captures structural snapshots of dynamic

172 processes, this variability might reflect the ensemble of $\mathrm{T}_{4} \mathrm{SS}_{\mathrm{pKM} 101}$ visualized at different stages of assembly or

173 activation.

175 Visualization of the in situ IMC. Next, we refined the structure of the IMC using class averages of machines

176 with detectable OMCC and IMC subassemblies (Figs. 2A, B, S2). Notable features of the IMC include a distinct

177 collar surrounding the central stalk, which in top-down view presents as six knobs arranged in a ring of $155 \AA$.

178 The collar was flanked by six protrusions or 'bumps' that were clearly distinct from the IM density (Fig. 2B, D).

179 At the cytoplasmic face of the IM, the IMC was dominated by two side-by-side inverted ' $\mathrm{V}$ ' structures with

180 apices embedded in the IM and 'arms' projecting $\sim 80 \AA$ into the cytoplasm (Fig. 2B). In end-on view, six V

181 structures clearly form two concentric circles, the outer arms of the V's configured as a knobbed ring of $\sim 225 \AA$

182 in diameter and the inner arms joining together as a central hexameric ring with an outer diameter of $\sim 60 \AA$ and a

183 lumen of $\sim 45 \AA$. As for the periplasmic collar, the 6-fold symmetry of the concentric rings was readily visible

184 among the class average images without symmetry imposed (Fig. S2). The structure was resolved further by

185 imposing a 6-fold symmetry during refinement (Figs. 2B-D, S2).

187 The structure of the pKM101 IMC bears striking similarities to IMCs associated with the F plasmid-encoded Tra 188 and L. pneumophila Dot/Icm systems, whose structures also were solved by in situ CryoET (Fig. 3A-C) $(16,17)$. 
Most notably, the cytoplasmic complexes of all three systems appear as side-by-side V's in side view and as outer knobbed and inner continuous rings of similar sizes in end-on view. In studies of the F and Dot/Icm machines, structural analyses of mutant machines deleted of each of the T4SS ATPases, coupled with density tracing of a

192 GFP moiety fused to a VirB4 homolog, established that the V structures correspond to dimers of VirB4-like

193 ATPases $(16,17)$. The cytoplasmic complexes of the F plasmid and Dot/Icm systems therefore consist primarily

194 of VirB4 subunits arranged as a central hexamer of dimers at the base of the translocation channel. The IMC of

195 the H. pylori Cag T4SS is architecturally more complex than the IMCs of the F plasmid-encoded and Dot/Icm

196 systems, yet VirB4-like Cag $\beta$ is similarly configured as a hexamer of dimers at the Cag channel entrance (18).

The pKM101 IMC visualized here differs remarkably from that associated with the in vitro $\mathrm{VirB}_{3-10}$ structure

(Figs. 2B, C, 3A, 3Di-iii) (6). Most notably, the pKM101 IMC is highly symmetric in its overall 6-fold symmetrical periplasmic collar and cytoplasmic complex. The $\operatorname{VirB}_{3-10} \mathrm{IMC}$ is asymmetric and dominated by sideby-side barrel complexes, which consist at least partly of the VirB4 homolog TrwK as shown by gold labeling (6). homohexamer, and results of stoichiometric analyses showing that the $\mathrm{VirB}_{3-10}$ complex is composed of 12 copies of $\operatorname{TrwK}_{\mathrm{B} 4}(6,25)$.

The pKM101 cytoplasmic complex is dominated by VirB4-like TraB. To define contributions of VirB4-like TraB to the $\mathrm{T}_{4} \mathrm{SS}_{\mathrm{pKM} 101}$, we imaged $\Delta \operatorname{traB}_{B 4}$ mutant machines (100 machines from 254 tomographic reconstructions) (Fig. S5). We previously reported that $\operatorname{traB}_{B 4}$ expression in trans fully complements a $\Delta \operatorname{tra} B_{B 4}$ mutation, confirming that the mutation is nonpolar on downstream gene expression (8). Subvolume class averages of the $\Delta \operatorname{traB}_{B 4}$ mutant machines consisted of the OMCC without associated IMC densities (Fig. S5). Most notably, the $\Delta t r a B_{B 4}$ machines lacked cytoplasmic densities dominated by the concentric hexameric rings (Fig. 4B, S5), indicating that $\mathrm{TraB}_{\mathrm{B} 4}$ adopts the same hexamer-of-dimer architectures observed for VirB4 homologs associated with the F plasmid-encoded, Dot/Icm, and Cag T4SSs (16-18). This architecture is compatible with 
216 a membrane ATPase with a hexamer-of-dimer configuration. The VirB4 ATPases are arranged so that their N-

217 terminal domains (NTDs) associate tightly with the IM $(28,29)$, and C-terminal domains (CTDs) consisting of

218 RecA-like $\alpha / \beta$ structural folds extend into the cytoplasm $(30,31)$. An atomic structure of the CTD of a VirB4

219 homolog fitted optimally within densities comprising the proximal halves of the V arms (Fig. 4Eiii), which lends

220 further support to a conclusion that visualized hexamer of dimer densities are composed of $\operatorname{TraB}_{\mathrm{B} 4}$.

IMC densities other than the inverted V's also were missing in $\Delta t r a B_{B 4}$ mutant machines, including the

223 periplasmic stalk and surrounding collar and 'bumps' (Figs. 4B, S5). VirB4 homologs associate peripherally with

224 the IM (32) or at most possess small periplasmic domains (29), arguing against appreciable contributions of

$225 \mathrm{TraB}_{\mathrm{B} 4}$ to the observed collar or stalk densities. However, several IMC subunits are strong candidates for

226 constituting these densities. These include periplasmic linker domains of VirB10-like TraF and VirB5-like TraC,

227 which are likely components of the stalk (6). VirB8-like TraE is a strong candidate for the central collar, as

228 supported by a recent CryoEM structure showing that purified $\operatorname{TraE}_{\mathrm{B} 8}$ assembles as a homohexamer with

229 dimensions matching those of the collar visualized in situ (33). Here, we also determined that an atomic structure

230 of the periplasmic domain of $\operatorname{TraE}_{\mathrm{B} 8}$ (34) also fits well within each lobe of the in situ collar (Fig. 4Eii). Finally,

231 like other VirB6 subunits (35), $\operatorname{TraD}_{\mathrm{B} 6}$ has a large central periplasmic domain that likely also contributes to one or

232 more of the periplasmic densities. The absence of discernible periplasmic densities in the $\Delta$ traB mutant machines

233 suggests that $\mathrm{TraB}_{\mathrm{B} 4}-\mathrm{IMC}$ subunit contacts are necessary for stable assembly of the IMC. In line with this

234 proposal, numerous studies have presented evidence that VirB4-like subunits form a network of stabilizing

235 interactions with IMC constituents including homologs of VirB3, VirB5, VirB8, and VirB10 (8, 36-39).

237 Deletions of the TraJ or TraG ATPases do not detectably alter the in situ T4SS $_{\mathbf{p K M 1 0 1}}$. Nearly all T4SSs

238 require a VirD4-like ATPase, which serves to recruit and deliver secretion substrates into the transfer channel.

239 Designated as type IV coupling proteins (T4CPs) or substrate receptors, VirD4 subunits are members of the

240 SpoIIIE/FtsK superfamily of motor translocases $(40,41)$. To determine if VirD4-like TraJ contributes to densities

241 of the $\mathrm{T}_{4} \mathrm{SS}_{\mathrm{pKM} 101}$, we imaged $\Delta \operatorname{traJ}_{D 4}$ mutant machines (183 machines from 430 tomographic reconstructions).

242 As observed with the WT machines, subvolume averaging yielded classes of $\Delta \operatorname{traJ}_{D 4}$ mutant machines exhibiting 
243 only the OMCC or both the OMCC and IMC densities (Fig. S5). A refined structure generated from the latter

244 classes showed no distortions compared to WT machines, insofar as the OMCC, periplasmic collar and central

245 stalk, and cytoplasmic V structures were clearly evident (Fig. 4C). TraJ $\mathrm{D}_{\mathrm{D} 4}$ thus does not contribute detectably to

246 the in situ $\mathrm{T}_{4} \mathrm{SS}_{\mathrm{pKM} 101}$ structure. The F plasmid-encoded Tra and L. pneumophila Dot/Icm machines similarly

247 were unaltered upon deletion of their respective VirD4 receptors $(16,17)$. In a recently-updated in vitro structure

248 of the R388-encoded T4SS, densities thought to correspond to one or two dimers of VirD4-like TrwB were shown

249 to be integrated between the side-by-side VirB4 barrels (13). However, the in situ architecture of the $\Delta \operatorname{traJ}_{D 4}$

250 mutant machines, together with evidence that VirD4-like subunits assemble as homohexamers (40, 42) and

251 engage with T4SS channels only when activated by intracellular signals such as substrate binding and ATP

252 hydrolysis (43-47), suggests that the in vitro $\mathrm{VirB}_{3-10} / \mathrm{VirD} 4$ complexes might represent transition-state structures.

254 Many T4SSs also require a third ATPase designated VirB11 for substrate transfer and pilus production $(37,48)$.

255 VirB11 ATPases assemble as homohexamers that co-fractionate with the cytoplasm and IM, the latter presumably 256 in association with the T4SS $(16,49-51)$. To determine if $\mathrm{TraG}_{\mathrm{B} 11}$ contributes to the visualized $\mathrm{IMC}_{\mathrm{pKM} 101}$, we

257 imaged $\Delta \operatorname{tra} G_{B 11}$ mutant machines (257 machines from 537 tomograms). We were unable to detect any density

258 losses in the cytoplasmic complex of the $\Delta \operatorname{tra} G$ mutant machine compared with the WT machine (Fig. 4). This

259 suggests that $\mathrm{TraG}_{\mathrm{B} 11}$ might associate dynamically with the $\mathrm{T}_{4} \mathrm{SS}_{\mathrm{PKM} 101}$, as shown previously for VirB11-like

260 DotB in the L. pneumophila Dot/Icm system (16). In that system, detection of $\operatorname{DotB}_{\mathrm{B} 11}$ at the base of DotO $\mathrm{B}_{\mathrm{B} 4}$

261 required deployment of a mutant form of DotB $\mathrm{B}_{\mathrm{B} 11}$ capable of binding but not hydrolyzing ATP (16).

Interestingly, we also observed that the $\Delta \operatorname{tra} G_{B 11}$ mutant machines exhibited structural aberrations compared with

264 the WT machines that were suggestive of profound effects of $\mathrm{TraG}_{\mathrm{B} 11}$ docking on $\mathrm{T}_{4} \mathrm{SS}_{\mathrm{pKM} 101}$ channel

265 architecture. Recall that OMCCs were associated with IMC densities in only $\sim 50 \%$ of the subvolume class

266 averages of WT machines. In striking contrast, in $\Delta t r a G_{B 11}$ machines, $\sim 85 \%$ of visualized OMCCs were

267 associated with IMC densities. Furthermore, the IMC densities were more clearly defined for the $\Delta \operatorname{tra} G_{B 11}$ mutant 268 machines compared with WT machines, and notably the central stalks were considerably elongated (Figs. 4, S5).

269 These findings suggest that $\operatorname{TraG}_{\mathrm{B} 11}$ plays an important role in regulating the conformational status of the central 
270 stalk and IMC. Previous work has presented evidence that VirB11 functions as a switch to regulate pilus-

271 biogenesis vs DNA-transport modes of action in the R388-encoded system (52). Furthermore, a recent in situ

272 CryoET study documented structural changes in the $\mathrm{IM}$ upon binding of DotB $\mathrm{B}_{\mathrm{B} 11}$ to DotO$_{\mathrm{B} 4}$ consistent with a role

273 for this ATPase in opening of the IM channel in the L. pneumophila Dot/Icm system (21). It is enticing to

274 propose that $\mathrm{TraG}_{\mathrm{B} 11}$ binding with $\mathrm{TraB}_{\mathrm{B} 4}$ might similarly open the IM channel of the T4SS $\mathrm{pKM} 101_{\text {and also induce }}$

275 structural transitions in the central stalk of importance for substrate passage to the cell exterior. Further studies

276 examining the structural consequences of $\operatorname{TraG}_{\mathrm{B} 11}$ and $\operatorname{TraJ}_{\mathrm{D} 4}$ docking with the $\mathrm{T}_{4} \mathrm{SS}_{\mathrm{PKM} 101}$ are clearly warranted.

278 Summary. CryoET has emerged as a valuable complementary approach to single-particle CryoEM studies of

279 bacterial secretion nanomachines (53). Although current resolutions achievable with CryoET are lower than

280 CryoEM, structural definition of machines in their native contexts enables i) validation of architectural features

281 observed in vitro, ii) assessments of machine structural variability within and between species, and iii)

282 visualization of dynamic aspects of machine biogenesis and function $(3,53,54)$. Here, we presented the first in

283 situ structures of a 'minimized' T4SS elaborated by the model conjugative plasmid pKM101. We showed that the

284 in vivo $\mathrm{T}_{4} \mathrm{SS}_{\mathrm{pKM} 101}$ consists of two large substructures, the OMCC and IMC, and that the former resembles

285 structures of equivalent complexes solved in vitro $(4,7,24)$. We further identified specific OM contacts, supplied

286 evidence for OM remodeling during $\mathrm{T}_{4} \mathrm{SS}_{\mathrm{pKM} 101}$ biogenesis, and visualized a central stalk similar to that detected

287 in the isolated $\mathrm{VirB}_{3-10}$ complex (6). We further gained evidence for contributions of TraGB11 to assembly or

288 configuration of the IMC and central stalk, in agreement with recent findings for DotB ${ }_{\mathrm{B} 11}$ in the Dot/Icm system

289 (21). Most importantly, we showed that $\operatorname{TraB}_{\mathrm{B} 4}$ assembles as a central hexamer of dimers, an oligomeric

290 conformation similar to those of VirB4 homologs associated with the F-encoded Tra, L. pneumophila Dot/Icm

291 and H. pylori Cag T4SSs (16-18). IMCs of T4SSs are characteristically highly unstable and difficult to purify in

292 the presence of detergents $(4,9-12,24)$, raising the possibility that the side-by-side barrel arrangement described

293 for Trw $\mathrm{K}_{\mathrm{B} 4}$ in the $\mathrm{VirB}_{3-10}$ complex (6) might be a structural artefact of machine purification.

295 Together with previous biochemical and structural data $(21,36,37,45,55)$, our findings support a model in which

296 VirB4 ATPases play critical roles in several key steps of T4SS biogenesis, as depicted in Fig. 5. Stage I: The 
297 intrinsically-stable OMCC assembles without contributions by VirB4 or other ATPases. This stage I reaction is

298 supported by our in situ evidence that the pKM101 OMCC assembles in the absence of associated IMC densities

299 (Fig. S2). Stage II: The OMCC recruits VirB4 through previously-identified interactions between the ATPase

300 and the cell-envelope-spanning VirB10 subunit (37), and then VirB4 recruits or stabilizes other IMC components

301 including VirB3, VirB5, VirB6, and VirB8 to yield the IMC. This stage II reaction is supported by our findings

302 that $\operatorname{TraB}_{\mathrm{B} 4}$ is required for detection of the periplasmic densities including the collar, flanking 'bumps', and

303 central stalk structures (Fig. 1). Stage III: Upon receipt of an unknown signal, VirB4 recruits the spatially-

304 dynamic VirB11 ATPase, which in turn induces structural changes in the stalk and IMC (Fig. 4) of postulated

305 importance for the transition from a pilus-generating machine to substrate translocation channel $(21,52)$. Stage

306 IV: Finally, upon substrate docking, the VirD4 substrate receptor binds VirB4, and the three ATPases coordinate

307 substrate delivery through the lumen of the VirB4 hexamer and into the T4SS channel $(37,48)$. The proposed

308 stage III and IV reactions are supported by our analyses of the $\Delta t r a G_{B 11}$ and $\Delta t r a J_{D 4}$ mutant machines and recent

309 findings for the Dot/Icm system $(16,21)$. However, it is also clear that further in situ studies aimed at visualizing

310 T4SSs with stably-engaged VirB11 and VirD4 subunits, or of WT T4SSs in the act of translocating DNA or other

311 substrates to recipient cells, will provide valuable new insights into structural transitions necessary for machine

312 activation. 


\section{MATERIALS AND METHODS}

315 Strains and growth conditions. Bacterial strains, plasmids, and oligonucleotides used in this study are listed in

316 Table S1. E. coli strains were grown at $37^{\circ} \mathrm{C}$ in Luria Bertani (LB) agar or broth supplemented with appropriate

317 antibiotics (kanamycin, $100 \mu \mathrm{g} \mathrm{ml}^{-1}$; spectinomycin, $100 \mu \mathrm{g} \mathrm{ml}^{-1}$; gentamycin, $10 \mu \mathrm{g} \mathrm{ml}^{-1}$ ). Minicells from E. coli 318 strain UU2834 were used for all of the CryoET studies.

320 Conjugation assays. E. coli MG1655 strains carrying pKM101 or mutant variants were used as donors to

321 transfer the plasmids into UU2834 recipients. Strains containing the pKM101 mutants also harbored a

322 complementing plasmid. Overnight cultures of donor and recipient cells grown in presence of the appropriate

323 antibiotics at $37^{\circ} \mathrm{C}$ were diluted 1:1,000 in fresh LB media, and incubated with shaking for $1.5 \mathrm{~h}$. When needed,

324 cells were induced with arabinose ( $0.2 \%$ final concentration) and incubated with shaking for another $1.5 \mathrm{~h}$. Equal

325 volumes $(50 \mu \mathrm{l})$ of donor and recipient cell cultures were mixed and incubated for $3 \mathrm{~h}$ at $37^{\circ} \mathrm{C}$. Mating mixtures

326 were serially diluted and plated onto LB agar containing antibiotics selective for transconjugants (Tc's). Plasmid-

327 carrying UU2834 strains were verified for the presence or absence of tra genes of interest by PCR. For matings to 328 assess minicell donor capacity, minicells were spotted onto a nitrocellulose filter disc alone or with MC4100 rif $^{r}$

329 recipient cells, and the mating mixes were incubated at $37^{\circ} \mathrm{C}$ for $1 \mathrm{~h}$. Discs were suspended in LB, serially diluted 330 and plated on LB agar plates containing appropriate antibiotics selective for donors (to confirm absence of viable 331 donor cells), recipients, or transconjugants (Tc's). Because minicells are nonviable, the frequency of transfer is 332 reported as Tc's/recipient. Matings were performed two times in triplicate, and results are presented as the mean 333 frequency of transfer with the standard error of mean (SEM) shown.

335 Isolation of minicells. E. coli minicells were enriched essentially as described previously (56, 57). E. coli $336 \mathrm{UU} 2834$ harboring pKM101 or variants were grown overnight at $37^{\circ} \mathrm{C}$ in $\mathrm{LB}$ in the presence of spectinomycin, 337 and then subcultured $(1: 100)$ in fresh $\mathrm{LB}$ devoid of antibiotics at $37^{\circ} \mathrm{C}$ to an $\mathrm{OD}_{600}=0.5$. Anucleate minicells were 338 selectively enriched by centrifugation at 2,000xg for $10 \mathrm{~min}$ at room temperature to pellet rod-shaped cells. Next, 339 the supernatant was centrifuged at $10,000 \mathrm{xg}$ for $10 \mathrm{~min}$ to pellet the minicells. The minicells were resuspended in 340 fresh $\mathrm{LB}$ and incubated at $37^{\circ} \mathrm{C}$ with gentle shaking for $45 \mathrm{~min}$ to reinitiate cell growth. Ceftriaxone (final 
341 concentration $100 \mu \mathrm{g} \mathrm{ml}^{-1}$ ) was added to the minicell preparation to lyse growing cells, and the culture was further

342 incubated at $37^{\circ} \mathrm{C}$ for $1 \mathrm{hr}$. The preparation was centrifuged at $400 \mathrm{xg}$ for $10 \mathrm{~min}$ to remove dead cells and debris.

343 The supernatant was centrifuged at 10,000xg for $10 \mathrm{~min}$ to harvest the minicells. The minicells were washed twice

344 in fresh LB, filtered through a $0.45 \mu \mathrm{m}$ filter (Millipore) and then used for the mating assay. To minimize possible

345 breakage of the pKM101-encoded pilus and to concentrate minicells for CryoET analyses, UU2834 strains were

346 grown overnight on LB agar plates at $37^{\circ} \mathrm{C}$. Cells were gently scraped from the plate surface with an "L" shaped

347 rod and resuspended in phosphate-buffered saline (PBS). The cell suspension was centrifuged twice at 1,000xg for

$3483 \mathrm{~min}$ to remove intact cells and the supernatant was then centrifuged at 10,000xg for 20 min and the minicell

349 pellet was resuspended in PBS for preparation of grids for CryoET.

351 Preparation of frozen-hydrated specimens. Minicells resuspended in PBS were mixed with 10 nm diameter

352 colloidal gold particles (Aurion BSA Gold Tracer, $10 \mathrm{~nm}$ ) and deposited onto freshly glow-discharged, holey

353 carbon grids (Quantifoil R2/1 200 mesh copper) for 1 min. After blotting the grids with filter paper, they were

354 rapidly frozen in liquid ethane by using a gravity-driven plunger apparatus $(58,59)$.

356 Cryo-ET data collection and 3D reconstructions. Frozen-hydrated specimens were imaged and data were

357 processed using our previously established protocols $(12,28,41)$. Briefly, specimens were subjected to imaging at $358-170^{\circ} \mathrm{C}$ using a Polara G2 electron microscope (FEI Company) equipped with a field emission gun and a direct

359 detection device (Gatan K2 Summit). The microscope was operated at $300 \mathrm{kV}$ at a magnification of $15 \mathrm{~K}$,

360 resulting in an effective pixel size of $2.5 \AA$ at the specimen level (17). Tomographic package SerialEM (60) was

361 used to collect low-dose, single-axis tilt series with dose fractionation mode and a defocus at $\sim 6 \mu \mathrm{m}$ and a

362 cumulative dose of $\sim 60 \mathrm{e}^{-} / \AA^{2}$ distributed over 35 stacks. Each stack contains $\sim 8$ images. Each tilt series was

363 collected at angles from $-51^{\circ}$ to $51^{\circ}$ with $3^{\circ}$ fixed increments. We used Tomoauto (58) to expedite data

364 processing, which included drift correction of dose-fractionated data using Motioncorr (61) and assembly of

365 corrected sums into tilt series, automatic fiducial seed model generation, alignment and contrast transfer function

366 correction of tilt series by IMOD (62), and reconstruction of tilt series into tomograms by Tomo3D (63). Each

367 tomographic reconstruction was 3,710 by 3,838 by 2,400 pixels and $\sim 130 \mathrm{~Gb}$ in size. 
368 Subtomogram averaging and correspondence analysis. Tomographic package I3 (64) was used for

369 subtomogram analysis as described previously (65). A total of 837 T4SS $\mathrm{pKM} 101_{1}$ machines (400 x 400 x 400 voxels)

370 were visually identified and then extracted from 1781 cryo-tomographic reconstructions. Two of the three Euler

371 angles of each $\mathrm{T}_{4} \mathrm{SS}_{\mathrm{PKM} 101}$ machine were estimated based on the orientation of each particle in the cell envelope.

372 To accelerate image analysis, 4 x 4 x 4 binned subtomograms (100 x 100 x 100 voxels) were used for initial

373 alignment. The alignment proceeded iteratively, with each iteration consisting of three parts in which references

374 and classification masks were generated, subtomograms were aligned and classified, and, finally, class averages

375 were aligned to each other. At the initial iterations, classification mask was applied to include the whole machine

376 and non-T4SS particles were sorted out and removed. For analysis of the IMC, a mask was applied to the IMC

377 only, thus the T4SS particles that did not show IMC density were sorted out and the data set showing IMC was

378 used to further refine the IMC. Classification focusing on the OMCC displayed 14-fold symmetry; therefore, 14-

379 fold symmetry was imposed in the following processing to assist the initial alignment process. Classification

380 focusing on the IMC showed a 6-fold symmetry feature, and in the following processing 6-fold symmetry was

381 imposed to assist in sub-tomograms alignment. After multiple cycles of alignment and classification for $4 \times 4 \times 4$

382 binned sub-tomograms, 2 × 2 × 2 binned sub-tomograms was used for refinement. Fourier shell correlation (FSC)

383 between the two independent reconstructions was used to estimate the resolution of the averaged structures (see

384 Fig. S2).

385

386 3D visualization. IMOD was used to visualize the maps and generate 3D surface rendering of $E$. coli minicells.

387 UCSF Chimera (66) (http://www.rbvi.ucsf.edu/chimera) was used to visualize subtomogram averages in 3D and

388 for molecular modeling. The video clips for the supplemental videos were made by using UCSF Chimera and

389 further edited with iMovie.

391 Data availability. Density maps and coordinate data of the $\mathrm{T}_{4} \mathrm{SS}_{\mathrm{pKM} 101}$ machines determined by cryo-electron

392 tomography have been deposited in the Electron Microscopy Data Bank (EMDB) as EMD-24100 and EMD-

393 24098. The authors declare that all other data supporting the findings of this study are available within the paper

394 and its supplementary information files. 


\section{LEGEND FOR SUPPLEMENTARY MATERIALS.}

396 Table S1. List of strains, plasmids, and oligonucleotides used in these studies.

397 Fig. S1. Genes and encoded functions of "minimized" type IV secretion systems (T4SSs) in Gram-negative 398 species.

399 Fig. S2. Workflow for in situ CryoET.

400 Fig. S3. Detection of pKM101 pilus docked on E. coli outer membrane without underlying basal structures.

401 Fig. S4. Comparisons of the outer membrane core complexes (OMCCs) and stalks/channels of 'minimized' and 402 'expanded' T4SSs.

403 Fig. S5. Heterogeneity of the pKM101 T4SS machines revealed by class sub-volume averaging.

404 Movie S1. 3-D visualization of a tomographic reconstruction and the T4SS $\mathrm{pKM}_{\mathrm{P} 101}$ in E. coli minicells.

405 Movie S2. 3-D visualization of the $\mathrm{T}_{4} \mathrm{SS}_{\mathrm{pKM} 101}$ showing architectural features of the OMCC and IMC and its 406 comparison with purified $\mathrm{VirB}_{3-10}$ complex from R388.

408 ACKNOWLEDGEMENTS. B.H. was supported by McGovern Medical School start-up funds, the Welch 409 Foundation (AU-1953-20180324), NSF grant \#1902392, and NIH 1R35GM138301. P.J.C. was supported by NIH 410 1R35GM131892 and R01GM48746. B. H. and P.J.C were supported by NIH R21AI142378. We thank members 411 of the Christie and Hu labs for helpful discussions, and Dr. William Margolin for advice on minicell purification. 


\section{FIGURE LEGENDS}

414 Fig. 1. E. coli minicells carrying pKM101 encoded type IV secretion system $\left(\mathrm{T}_{4} \mathrm{SS}_{\mathrm{pKM} 101}\right)$ and in situ structure of

415 the outer membrane core complex $(\mathrm{OMCC})$ of $\mathrm{T}_{4} \mathrm{SS}_{\mathrm{pKM} 101}$ revealed by CryoET and subtomogram averaging. (Ai,

416 ii, iii) Tomographic slices from representative $E$. coli minicells showing T4SSs embedded between the outer

417 membrane (OM) and inner membrane (IM). pKM101 pili were associated with a few visualized T4SSs, although

418 pilus associated OM structures without any periplasmic densities. The T4SS and novel structures are marked with

419 yellow arrow. The boxed regions were magnified to show T4SS with and without associated pilus and also pilus

420 associated OM structures. (Aiv) A 3D surface view of the E. coli minicell in A iii showing T4SSs. (Bi) A central

421 slice of the averaged structure of the T4SS in the cell envelope. (Bii) After refinement, details of the OMCC are

422 visible. The widths and heights of O-layer and I-layer chambers are shown. (Biii) A cross-section view of the

423 region in Bii marked by a yellow arrow shows 14-fold symmetry of the OMCC. (C) 3D surface renderings of the

424 OMCC are shown in different views.

425 Fig. 2. In situ structure of the inner membrane complex (IMC) of pKM101 encoded type IV secretion system

426 (T4SS $_{\text {pKM101 }}$ ) revealed by CryoET and subtomogram averaging. (A) A central slice of the averaged structure of the

427 T4SS in the cell envelope. (B) After refinement, details of the IMC are visible. The stalk, collar and inverted "V"

428 structures along with the height of its arms are shown. (C) Cross-section views of the regions in B marked by

429 yellow arrows show 6-fold symmetry of the IMC. The collar exists as a hexameric ring-like structure around the

430 central stalk. (D) 3D surface renderings of the IMC are shown in different views.

432 Fig. 3. Comparison of the inner membrane complexes (IMCs) solved by CryoET and single particle analysis. (A,

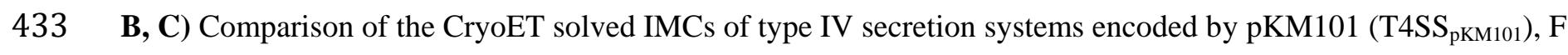

434 plasmid (T4SS $\mathrm{pED} 208)$ and L. pneumophila $\left(\mathrm{T}_{4} \mathrm{SS}_{\mathrm{Dot} / \mathrm{Icm}}\right)$. 3D surface renderings showing 6-fold symmetric IMCs

435 marked by the hexamer of dimer arrangement of $\mathrm{VirB}_{4}$ homologs. (Di) A central slice of the averaged structure of

436 the purified VirB $_{3-10}$ substructure encoded by plasmid R388. (Dii) A cross-section view of the region in Di

437 marked by a yellow arrow shows 2-fold symmetry exist in IMC of purified VirB ${ }_{3-10}$. (Diii) The surface rendering 
438 of the VirB $_{3-10}$ substructure highlighting the IMC with side-by-side two hexamers of the TrwK/VirB4 ATPase

439 (pink-shaded). F plasmid EMD: 9344 and 9347; Dot/Icm EMD: 7611 and 7612; VirB 3-10 EMD:2567.

441 Fig. 4. Architecture of $\mathrm{T}_{4} \mathrm{SS}_{\mathrm{pKM} 101}$ mutant machines from strains lacking one of the Tra ATPases and comparison

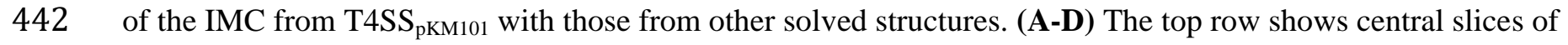
443 the averaged structures from strains carrying native pKM101 or the $\Delta \operatorname{traB}, \Delta \operatorname{traJ}$ and $\Delta \operatorname{traG}$ mutant plasmids. The 444 second row shows the refined IMCs of the corresponding strains. (Ei) A 3D surface rendering of the IMC of $445 \mathrm{~T}_{4} \mathrm{SS}_{\mathrm{pKM} 101}$. (Eii) End-on view of the hexameric collar. A crystal structure of the periplasmic domain of pKM101446 encoded $\mathrm{TraE}_{\mathrm{B} 8}$ (PDB: 5I97) fits well in the lobe-like structure of the collar. (E iii) A crystal structure of the C447 terminal domain (CTD) of VirB4 ATPase from Thermoanaerobacter pseudethanolicus (PDB:4AG5) fits well in 448 one of the arms of the TraB hexamer of dimers.

450 Fig. 5. A working model depicting contributions of the VirB4 ATPases to early stages of T4SS assembly and 451 substrate routing. Stages: I) The OMCC assembles as an intrinsically-stable substructure independently of 452 contributions by the T4SS ATPases. II) VirB4 is recruited to the OMCC through contacts with the N-terminal 453 cytoplasmic and IM transmembrane domains of VirB10. VirB4 assembles as a hexamer of dimers at the 454 cytoplasmic face of the IM, where it recruits and stabilizes other IMC and stalk constituents. III) VirB4 serves as 455 a docking site for the spatially-dynamic VirB11 ATPase; docked VirB11 regulates structural transitions necessary 456 for channel activation. IV) The VirD4-like substrate receptor or T4CP (type IV coupling protein) oligomerizes 457 and hydrolyzes ATP in response to binding of DNA or protein substrates; the VirD4 - substrate complex engages 458 with and activates the T4SS channel. The ATPase energy center composed of the VirB4, VirB11, and VirD4 459 ATPases coordinate processing and delivery of secretion substrates through the central lumen of VirB4 and into 460 the translocation channel $(37,48)$. Red lightning bolts denote signals, such as substrate binding and VirD4 ATP 461 hydrolysis and establishment of T4SS contact with target cell, that activate the T4SS for transfer. Relaxase, the 462 enzyme responsible for nicking the DNA strand (T-strand) destined for transfer; relaxosome, the assemblage of 463 processing proteins at the origin-of-transfer (oriT) sequence that are responsible for nicking and unwinding the T464 strand for transfer (see (2)). 


\section{REFERENCES}

466 1. Grohmann E, Christie PJ, Waksman G, Backert S. 2018. Type IV secretion in Gram-negative and Gram467 positive bacteria. Mol Microbiol 107:455-471.

468 2. Waksman G. 2019. From conjugation to T4S systems in Gram-negative bacteria: a mechanistic biology 469 perspective. EMBO Rep 20:47012. doi: 10.15252/embr.201847012.

470 3. Costa TRD, Harb L, Khara P, Zeng L, Hu B, Christie PJ. 2021. Type IV secretion systems: Advances in 471 structure, function, and activation. Mol Microbiol 115:436-452.

472 4. Fronzes R, Schafer E, Wang L, Saibil HR, Orlova EV, Waksman G. 2009. Structure of a type IV 473 secretion system core complex. Science 323:266-268.

474 5. Christie PJ, Atmakuri K, Krishnamoorthy V, Jakubowski S, Cascales E. 2005. Biogenesis, architecture, 475 and function of bacterial type IV secretion systems. Annu Rev Microbiol 59:451-485.

476 6. Low HH, Gubellini F, Rivera-Calzada A, Braun N, Connery S, Dujeancourt A, Lu F, Redzej A, Fronzes 477 R, Orlova EV, Waksman G. 2014. Structure of a type IV secretion system. Nature 508:550-553.

478 7. Chandran V, Fronzes R, Duquerroy S, Cronin N, Navaza J, Waksman G. 2009. Structure of the outer 479 membrane complex of a type IV secretion system. Nature 462:1011-1015.

480 8. Gordon JE, Costa TRD, Patel RS, Gonzalez-Rivera C, Sarkar MK, Orlova EV, Waksman G, Christie PJ. 4812017 . Use of chimeric type IV secretion systems to define contributions of outer membrane 482 subassemblies for contact-dependent translocation. Mol Microbiol 105:273-293.

483 9. Sgro GG, Costa TR, Cenens W, Souza DP, Cassago A, Oliverira LD, Salinas RK, Portugal RV, Farah 484 DS, Waksman G. 2018. CryoEM structure of the core complex of a bacterial killing type IV secretion 485 system. Nat Microbiol 3:1429-1440. doi: 10.1038/s41564-018-0262-z.

486 10. Chung JM, Sheedlo MJ, Campbell AM, Sawhney N, Frick-Cheng AE, Lacy DB, Cover TL, Ohi MD. 487 2019. Structure of the Helicobacter pylori Cag type IV secretion system. Elife 8:e47644. doi: $488 \quad 10.7554 /$ eLife.47644.

489 11. Durie CL, Sheedlo MJ, Chung JM, Byrne BG, Su M, Knight T, Swanson M, Lacy DB, Ohi MD. 2020. 490 Structural analysis of the Legionella pneumophila Dot/Icm type IV secretion system core complex. Elife 491 9:e59530. doi: 10.7554/eLife.59530. 
492 12. Sheedlo MJ, Chung JM, Sawhney N, Durie CL, Cover TL, Ohi MD, Lacy DB. 2020. Cryo-EM reveals 493 species-specific components within the Helicobacter pylori Cag type IV secretion system core complex. $494 \quad$ Elife 9:e59495. doi: 10.7554/eLife.59495

495 13. Redzej A, Ukleja M, Connery S, Trokter M, Felisberto-Rodrigues C, Cryar A, Thalassinos K, Hayward 496 RD, Orlova EV, Waksman G. 2017. Structure of a VirD4 coupling protein bound to a VirB type IV 497 secretion machinery. EMBO J doi:10.15252/embj.201796629.

498 14. Ghosal D, Chang YW, Jeong KC, Vogel JP, Jensen GJ. 2017. In situ structure of the Legionella Dot/Icm 499 type IV secretion system by electron cryotomography. EMBO Rep 18:726-732.

500 15. Chang YW, Shaffer CL, Rettberg LA, Ghosal D, Jensen GJ. 2018. In vivo structures of the Helicobacter $501 \quad$ pylori cag type IV secretion system. Cell Rep 23:673-681.

502 16. Chetrit D, Hu B, Christie PJ, Roy CR, Liu J. 2018. A unique cytoplasmic ATPase complex defines the 503 Legionella pneumophila type IV secretion channel. Nat Microbiol 3:678-686.

504 17. Hu B, Khara P, Christie PJ. 2019. Structural bases for F plasmid conjugation and F pilus biogenesis in $505 \quad$ Escherichia coli. Proc Natl Acad Sci U S A 116:14222-14227.

506 18. Hu B, Khara P, Song L, Lin AS, Frick-Cheng AE, Harvey ML, Cover TL, Christie PJ. 2019. In situ 507 molecular architecture of the Helicobacter pylori Cag type IV secretion system. mBio 10(3):e00849-19. 508 doi: $10.1128 / \mathrm{mBio} .00849-19$.

509 19. Ghosal D, Jeong KC, Chang YW, Gyore J, Teng L, Gardner A, Vogel JP, Jensen GJ. 2019. Molecular $510 \quad$ architecture, polar targeting and biogenesis of the Legionella Dot/Icm T4SS. Nat Microbiol 4:1173-1182.

$51120 . \quad$ Chetrit D, Park D, Hu B, Liu J, Roy CR. 2020. Applying live cell imaging and cryo-electron tomography 512 to resolve spatiotemporal features of the Legionella pneumophila Dot/Icm secretion system. J Vis Exp 513 doi:10.3791/60693.

514 21. Park D, Chetrit D, Hu B, Roy CR, Liu J. 2020. Analysis of Dot/Icm type IVB secretion system 515 subassemblies by cryoelectron tomography reveals conformational changes induced by DotB binding. 516 mBio 11:e03328-19. doi: 10.1128/mBio.03328-19.

517 22. Farley MM, Hu B, Margolin W, Liu J. 2016. Minicells, back in fashion. J Bacteriol 198:1186-1195. 518 23. Bradley DE. 1980. Morphological and serological relationships of conjugative pili. Plasmid 4:155-169. 
519 24. Rivera-Calzada A, Fronzes R, Savva CG, Chandran V, Lian PW, Laeremans T, Pardon E, Steyaert J,

520 Remaut H, Waksman G, Orlova EV. 2013. Structure of a bacterial type IV secretion core complex at 521 subnanometre resolution. EMBO J 32:1195-1204.

522 25. Pena A, Matilla I, Martin-Benito J, Valpuesta JM, Carrascosa JL, de la Cruz F, Cabezon E, Arechaga I.

523 2012. The hexameric structure of a conjugative VirB4 protein ATPase provides new insights for a

524 functional and phylogenetic relationship with DNA translocases. J Biol Chem 287:39925-39932.

525 26. Durand E, Oomen C, Waksman G. 2010. Biochemical dissection of the ATPase TraB, the VirB4

526 homologue of the Escherichia coli pKM101 conjugation machinery. J Bacteriol 192:2315-2323.

527 27. Durand E, Waksman G, Receveur-Brechot V. 2011. Structural insights into the membrane-extracted

528 dimeric form of the ATPase TraB from the Escherichia coli pKM101 conjugation system. BMC Struct

$529 \quad$ Biol 11:4. doi: 10.1186/1472-6807-11-4.

530 28. Arechaga I, Pena A, Zunzunegui S, del Carmen Fernandez-Alonso M, Rivas G, de la Cruz F. 2008.

531 ATPase activity and oligomeric state of TrwK, the VirB4 homologue of the plasmid R388 type IV

532 secretion system. J Bacteriol 190:5472-5479.

533 29. Dang TA, Christie PJ. 1997. The VirB4 ATPase of Agrobacterium tumefaciens is a cytoplasmic

534 membrane protein exposed at the periplasmic surface. J Bacteriol 179:453-462.

535 30. Middleton R, Sjolander K, Krishnamurthy N, Foley J, Zambryski P. 2005. Predicted hexameric structure 536 of the Agrobacterium VirB4 C terminus suggests VirB4 acts as a docking site during type IV secretion.

537 Proc Natl Acad Sci U S A 102:1685-1690.

538 31. Wallden K, Williams R, Yan J, Lian PW, Wang L, Thalassinos K, Orlova EV, Waksman G. 2012.

539 Structure of the VirB4 ATPase, alone and bound to the core complex of a type IV secretion system. Proc $540 \quad$ Natl Acad Sci USA 109:11348-11353.

541 32. Schandel KA, Muller MM, Webster RE. 1992. Localization of TraC, a protein involved in assembly of 542 the F conjugative pilus. J Bacteriol 174:3800-3806.

543 33. Casu B, Mary C, Sverzhinsky A, Fouillen A, Nanci A, Baron C. 2018. VirB8 homolog TraE from 544 plasmid pKM101 forms a hexameric ring structure and interacts with the VirB6 homolog TraD. Proc Natl $545 \quad$ Acad Sci U S A 115:5950-5955. 
546 34. Casu B, Smart J, Hancock MA, Smith M, Sygusch J, Baron C. 2016. Structural analysis and inhibition of 547 TraE from the pKM101 type IV secretion system. J Biol Chem 291:23817-23829.

548 35. Jakubowski SJ, Krishnamoorthy V, Cascales E, Christie PJ. 2004. Agrobacterium tumefaciens VirB6 549 domains direct the ordered export of a DNA substrate through a type IV secretion system. J Mol Biol $550 \quad 341: 961-77$.

551 36. Yuan Q, Carle A, Gao C, Sivanesan D, Aly KA, Hoppner C, Krall L, Domke N, Baron C. 2005. 552 Identification of the VirB4-VirB8-VirB5-VirB2 pilus assembly sequence of type IV secretion systems. J $553 \quad$ Biol Chem 280:26349-26359.

554 37. Atmakuri K, Cascales E, Christie PJ. 2004. Energetic components VirD4, VirB11 and VirB4 mediate 555

39. Mossey P, Hudacek A, Das A. 2010. Agrobacterium tumefaciens type IV secretion protein VirB3 is an inner membrane protein and requires VirB4, VirB7, and VirB8 for stabilization. J Bacteriol 192:2830-

40. Gomis-Ruth FX, Moncalian G, Perez-Luque R, Gonzalez A, Cabezon E, de la Cruz F, Coll M. 2001. The early DNA transfer reactions required for bacterial type IV secretion. Mol Microbiol 54:1199-1211. bacterial conjugation protein TrwB resembles ring helicases and F1- ATPase. Nature 409:637-641.

41. Gomis-Ruth FX, Sola M, de la Cruz F, Coll M. 2004. Coupling factors in macromolecular type-IV

\section{42. Hormaeche I, Alkorta I, Moro F, Valpuesta JM, Goni FM, De La Cruz F. 2002. Purification and}

43. Tato I, Zunzunegui S, de la Cruz F, Cabezon E. 2005. TrwB, the coupling protein involved in DNA properties of TrwB, a hexameric, ATP-binding integral membrane protein essential for R388 plasmid conjugation. J Biol Chem 277:46456-46462. 
572 44. Tato I, Matilla I, Arechaga I, Zunzunegui S, de la Cruz F, Cabezon E. 2007. The ATPase activity of the

573 DNA transporter TrwB is modulated by protein TrwA: implications for a common assembly mechanism $574 \quad$ of DNA translocating motors. J Biol Chem 282:25569-25576.

575 45. Cascales E, Atmakuri K, Sarkar MK, Christie PJ. 2013. DNA substrate-induced activation of the $576 \quad$ Agrobacterium VirB/VirD4 type IV secretion system. J Bacteriol 195:2691-2704.

577 46. Larrea D, de Paz HD, Arechaga I, de la Cruz F, Llosa M. 2013. Structural independence of conjugative 578 coupling protein TrwB from its type IV secretion machinery. Plasmid 70:146-153.

579 47. Li YG, Christie PJ. 2020. The TraK accessory factor activates substrate transfer through the pKM101

581 48. Cascales E, Christie PJ. 2004. Definition of a bacterial type IV secretion pathway for a DNA substrate. $582 \quad$ Science 304:1170-1173.

583 49. Rashkova S, Spudich GM, Christie PJ. 1997. Characterization of membrane and protein interaction

50. Yeo HJ, Savvides SN, Herr AB, Lanka E, Waksman G. 2000. Crystal structure of the hexameric traffic ATPase of the Helicobacter pylori type IV secretion system. Mol Cell 6:1461-1472.

587 51. Savvides SN, Yeo HJ, Beck MR, Blaesing F, Lurz R, Lanka E, Buhrdorf R, Fischer W, Haas R, Waksman G. 2003. VirB11 ATPases are dynamic hexameric assemblies: new insights into bacterial type

590 52. Ripoll-Rozada J, Zunzunegui S, de la Cruz F, Arechaga I, Cabezon E. 2013. Functional interactions of $591 \quad$ VirB11 traffic ATPases with VirB4 and VirD4 molecular motors in type IV secretion systems. J Bacteriol $592 \quad 195: 4195-4201$.

593 53. Oikonomou CM, Jensen GJ. 2019. Electron cryotomography of bacterial secretion systems. Microbiol $594 \quad$ Spectr 7:10.1128/microbiolspec.PSIB-0019-2018.

595 54. Oikonomou CM, Jensen GJ. 2017. Cellular electron cryotomography: Toward structural biology in situ. $596 \quad$ Annu Rev Biochem 86:873-896. 
597 55. Kerr JE, Christie PJ. 2010. Evidence for VirB4-mediated dislocation of membrane-integrated VirB2 pilin 598 during biogenesis of the Agrobacterium VirB/VirD4 type IV secretion system. J Bacteriol 192:4923-

5994934.

600 56. Park D, Lara-Tejero M, Waxham MN, Li W, Hu B, Galan JE, Liu J. 2018. Visualization of the type III secretion mediated Salmonella-host cell interface using cryo-electron tomography. Elife 7:e39514. doi:

57. Jivrajani M, Shrivastava N, Nivsarkar M. 2013. A combination approach for rapid and high yielding purification of bacterial minicells. J Microbiol Methods 92:340-343.

58. Morado DR, Hu B, Liu J. 2016. Using Tomoauto: A protocol for high-throughput automated cryoelectron tomography. J Vis Exp Jan 30;(107):e53608. doi: 10.3791/53608.

59. Hu B, Lara-Tejero M, Kong Q, Galan JE, Liu J. 2017. In situ molecular architecture of the Salmonella

60. Mastronarde DN. 2005. Automated electron microscope tomography using robust prediction of specimen movements. J Struct Biol 152:36-51.

611 61. Li X, Mooney P, Zheng S, Booth CR, Braunfeld MB, Gubbens S, Agard DA, Cheng Y. 2013. Electron counting and beam-induced motion correction enable near-atomic-resolution single-particle cryo-EM. Nat

614 62. Kremer JR, Mastronarde DN, McIntosh JR. 1996. Computer visualization of three-dimensional image data using IMOD. J Struct Biol 116:71-76.

63. Agulleiro JI, Fernandez JJ. 2015. Tomo3D 2.0--exploitation of advanced vector extensions (AVX) for 3D reconstruction. J Struct Biol 189:147-152. 
bioRxiv preprint doi: https://doi.org/10.1101/2021.08.19.457048; this version posted August 20, 2021. The copyright holder for this preprint (which was not certified by peer review) is the author/funder, who has granted bioRxiv a license to display the preprint in perpetuity. It is made available under aCC-BY-NC-ND 4.0 International license.

623 66. Pettersen EF, Goddard TD, Huang CC, Couch GS, Greenblatt DM, Meng EC, Ferrin TE. 2004. UCSF

624 Chimera--a visualization system for exploratory research and analysis. J Comp Chem 25:1605-1612.

625

626

627

628

629 


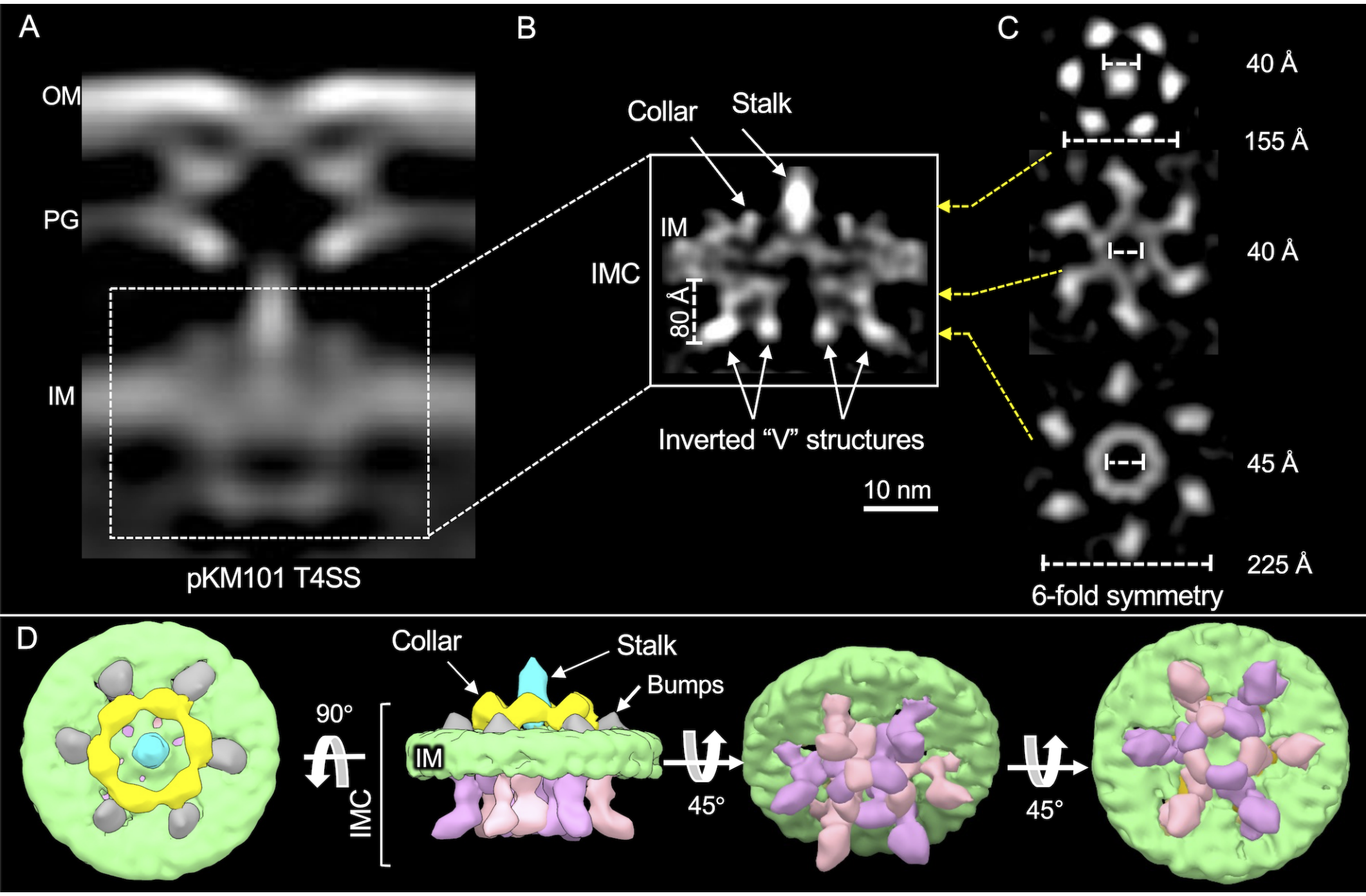



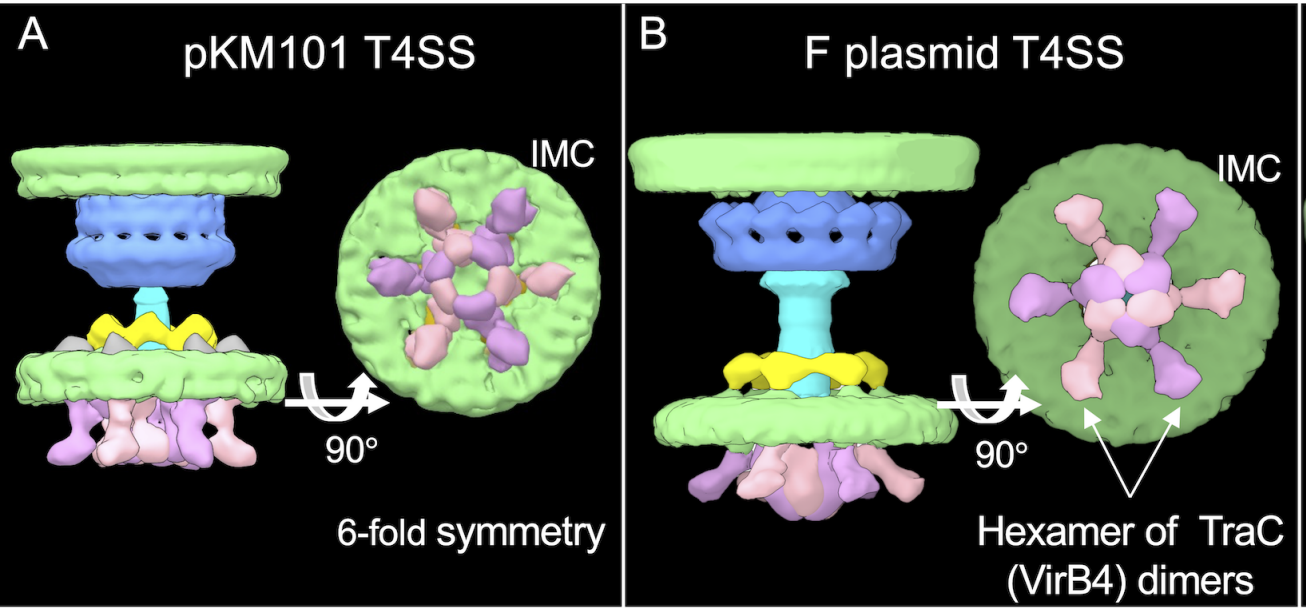

C L. pneumophila Dot/lam T4SS

D i

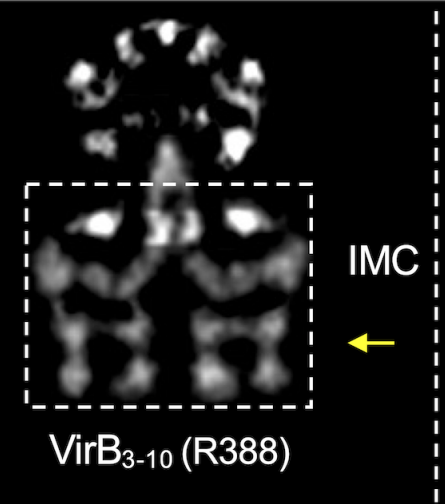

IMC

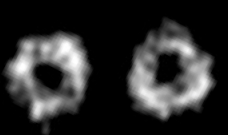

2-fold symmetry

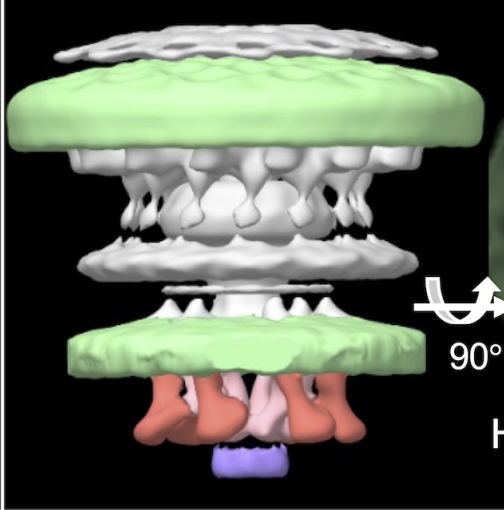

Hexamer of DotO (VirB4) dimers

\section{iii}

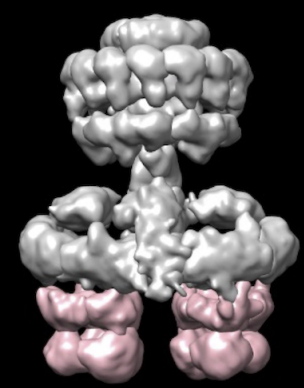

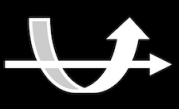

$90^{\circ}$

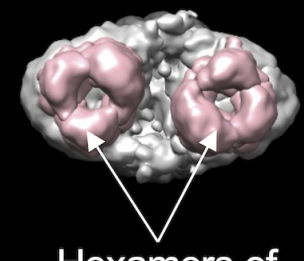

Hexamers of TrwK (VirB4) 
bioRxiv preprint doi: https://doi.org/10.1101/2021.08.19.457048; this version posted August 20, 2021. The copyright holder for this preprint (which was not certified by peer review) is the author/funder. who has granted bioRxiv a license to display the preprint in perpetuity. It is made

\section{A}

WT

B $\quad \Delta t r a B$
C $\Delta$ traJ
D $\Delta$ traG

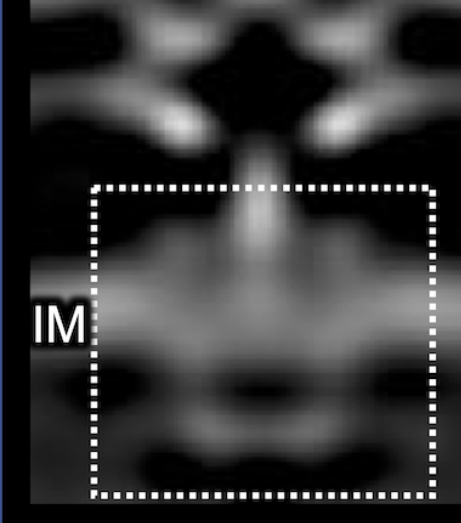

$\mathrm{E} \mathrm{i}$

IM $28 \times$ से

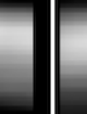

\section{(1)} ( 


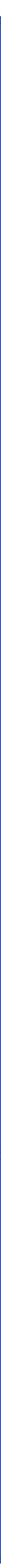

